\title{
Green Synthesis of Magnetite Nanoparticles using Banana Leaves
}

\author{
Ravindra D. Kale*, Sangeeta Barwar, Prerana Kane, Latika Bhatt
}

\begin{abstract}
In this study a simple, environment friendly and cost-effective method has been developed to synthesize metallic nanoparticles (NPs) from plant leaves. The study proposes that magnetite NPs can be synthesized using banana leaves as reducing agent. The effect of temperature and concentration of reducing agent on absorbance of solution was studied; optimization of the parameters was done using response surface methodology (RSM) as per central composite design (CCD). The results of $X$-ray diffraction (XRD) and Fourier Transform Infrared spectrometer (FTIR) indicated formation of iron oxide crystalline NPs in which polyols such as flavones, terpenoids and polysaccharides acted as reducing and capping agent. The characterization of synthesized magnetite NPs was also done through transmission electron microscopy (TEM), nanoparticle size analyzer and UV-Visible spectroscopy.
\end{abstract}

\section{KEYWORDS}

Nano Particle, Green Synthesis, Banana Leaves, Metal, Magnetite

\section{INTRODUCTION}

The advances in nano science and technology have made humans believe that it can ameliorate their current living standard (Priest 2006).

Department of Fibers and Textile Processing Technology

Institute of Chemical Technology

Nathalal Parekh Marg, Matunga (E)

Mumbai 400019

Maharashtra, India

*Corresponding author; email:

ravikale73pub@gmail.com
The specific characteristics of nanoparticles such as shape, size, and distribution can be utilized in various fields of applications, which make it a topic of interest for researchers globally (Zargar et al. 2011). The synthesis of $\mathrm{Fe}_{3} \mathrm{O}_{4}$ nanoparticles (MFeNPs) has been carried out because of its unique properties, such as being super paramagnetic (Mahdavian \& Mirrahimi 2010), biocompatible, biodegradable, and non-toxic to human beings (Wei et al. 2006; Zhao et al. 2009; Zhang et al. 2013). These properties of MFeNPs make them applicable in various areas, such as catalysis (Gwande and Varma 2013; Sharad et al. 2014), magnetic storage media (Terris \& Thomson 2005), biosensors (Kavitha et al. 2013), magnetic resonance imaging (MRI) (Haw et al. 2010; Qiao \& Gao 2009), and targeted drug delivery (Salem et al. 2015; Li et al. 2012; Wani et al. 2014).

MFeNPs can be fabricated through various methods such as sol-gel method (Lemine et al. 2012), solid state synthesis (Paiva et al. 2015), and flame spray synthesis (Kumfer et al. 2010). The chemical and physical methods of synthesis involve complicated procedures and are very time consuming while green synthesis method is much easier and safer to use. The green synthesis of NPs is a new scheme and researchers are still studying its outcomes. Few successful studies in synthesizing MFeNPs by using plant extract have been done before. For instance, fruit extract of Artemisia annua (Basavegowda et al. 2014), leaf extract of Perillafrutescens (Basavegowda et al. 2014), Tridaxprocumbens (Senthil \& Ramesh 2012) and caricaya papaya (Latha et al. 2014), peel extract of plantain (Venkateswarlu et al. 2013), and also seed extract of grape proanthocyanidin (Narayanan et al. 2012). However, only few studies have been done 
using marine plants for the synthesis of MFeNPs.

In the present work, Response Surface Methodology (RSM) is applied to see the effect of temperature and reducing agent concentration on the synthesis of MFeNPs using banana leaves as reducing agent thereby providing a green route of NPs synthesis. Synthesized NPs were then characterized using different techniques.

\section{RESULTS AND DISCUSSION Optimization}

The optimization of magnetite nanoparticles was performed by 13 experiments as per Table 1 and the values of each response was measured as solution absorbance at maximum wavelength of MFeNPs.

The quadratic model was checked, using the Design- Expert 7.0, trial version for ANOVA and the results are shown in Table 2. P-values were used as a tool to check the significance of each coefficient which also indicates the interaction strength of each parameter. In the present study, the F-value (28.44276) and P-values ( $p=$ 0.0009 ) indicated statistical significance of the obtained model. The degree of significance shows that the quadratic effects of temperature and concentration of reducing agent are significant; which means they can act as limiting factors and little variation in their value will alter the production rate. (Hamed et al. 2014) Value of adjusted $R^{2}=0.9196$ suggested that total variation of $91.96 \%$ of absorbance is attributed to the independent variables and only $8.04 \%$ cannot be explained by the model.

A second-order polynomial model (equation 1) was proposed to calculate the optimum levels of dependent and independent variables and to determine the maximum MFeNPssynthesis corresponding to theoptimum levels of temperature and concentration of reducing agent. By applying the multiple regression analysis on experimental data, the secondorder polynomial equation that defines predicted response (Absorbance) in terms of the independent variables (A, B) was obtained.

$$
\begin{gathered}
\text { Absorbance }=-0.19907+ \\
0.011524 \mathrm{~A}-0.074627 \mathrm{~B}+.00105160 \mathrm{AB}- \\
0.0000624497 \mathrm{~A}^{2}+0.00427503 \mathrm{~B}^{2}
\end{gathered}
$$

With the increase in temperature and concentration of reducing agent, absorbance of synthesized NPs increases (Fig. 1) which indicates increase in concentration of MFeNPs. According to the RSM, the results predicted by the model showed that the maximum absorbance can be achieved when the temperature and concentration of reducing agent are set at $70.47^{\circ} \mathrm{Cand} 2.5 \mathrm{~g} / 100 \mathrm{~mL}$ respectively. The maximum predicted value of absorbance obtained was 0.328339 . Under suggested conditions, the mean value of the absorbance was found to be 0.3278 , which is in agreement with the predicted value.

\section{Characterization}

UV -Visible spectral Analysis

Fig. 2 shows the UV-visible absorption spectrum of MFeNPs synthesized using banana leaves. The absorption peak at $360 \mathrm{~nm}$ indicates the presence of MFeNPs. (Chaki et al. 2015).

The appearance of green to grayish black colour of colloidal solution indicates the formation of MFeNPs with the increasing time (Fig. 3). (Latha et al. 2014) The colour changes arise due to the excitation of the surface plasma resonance (SPR) phenomenon typically of MFeNPs (Shankar et al. 2004). The optical absorption spectrum of MFeNPs depends on the particle size, shape, state of aggregation and the surrounding dielectric medium. (Petla et al. 2012)

\section{Particle size analysis}

Dynamic Light Scattering technique, TEM and XRD technique were used for particles size analysis of MFeNPs and shown in Figs. 4a-c and Table 3. From the light scattering technique, mean particle size of the nano particle was 31 nm. (Fig. 4a). The TEM images of MFeNPs synthesized by banana leaves showed spherical morphology in (Fig. 4b) and the size ranged from 45 to $120 \mathrm{~nm}$ with an average diameter of $72 \mathrm{~nm}$. (Fig. 4c) shows four peaks for the XRD pattern of MFeNPsat $35.7561^{\circ}$, 
$43.8031^{\circ}, 54.6528^{\circ}$ and $64.2351^{\circ}$ with (311), (400), (422) and (440) Miller indices respectively. (JCPDS card no. 00-003-0863) (Yen et al. 2016) DOI 10.1186/s11671-0161498-2. The synthesized particles had $19.22 \%$ of crystallinity which was calculated from the (400) peak. Thus, the MFeNPs had more amorphous structure. The reason being the fast-chemical reaction during synthesis which resulted in no clear reflection peak due to other crystalline phase, which might be present as impurity. Thus, the nano particles essentially consists of a binary mixture of the two spinel magnetic iron oxides, meaning magnetite-Fe3O4 and maghemite - $\gamma \mathrm{Fe} 2 \mathrm{O} 3$. \{Ref: Journal of Magnetism and Magnetic Materials 324 (2012) 1753-1757\}]

\begin{tabular}{|c|c|c|c|c|}
\hline \multirow{2}{*}{ Run } & Temperature & $\begin{array}{c}\text { Concentration of reducing agent } \\
\text { (gm/100 ml) }\end{array}$ & \multicolumn{2}{|c|}{ Absorbance at 360nm } \\
\cline { 2 - 5 } & $\left({ }^{\circ} \mathrm{C}\right)$ & & Experimental & Predicted \\
\hline 1 & 50 & 7.5 & 0.3001 & 0.296131 \\
\hline 2 & 75 & 7.5 & 0.5507 & 0.586254 \\
\hline 3 & 50 & 5 & 0.2361 & 0.217654 \\
\hline 4 & 75 & 5 & 0.5204 & 0.442052 \\
\hline 5 & 50 & 2.5 & 0.1702 & 0.192615 \\
\hline 6 & 100 & 2.5 & 0.4371 & 0.431898 \\
\hline 7 & 100 & 7.5 & 0.8299 & 0.798315 \\
\hline 8 & 75 & 5 & 0.4647 & 0.442052 \\
\hline 9 & 100 & 5 & 0.5516 & 0.588387 \\
\hline 10 & 75 & 5 & 0.4443 & 0.442052 \\
\hline 11 & 75 & 2.5 & 0.3685 & 0.351287 \\
\hline 12 & 75 & 5 & 0.3885 & 0.442052 \\
\hline 13 & 75 & 5 & 0.4107 & 0.442052 \\
\hline
\end{tabular}

Table 1: CCD experimental run of trials for synthesis of MFeNPs

\begin{tabular}{|c|c|c|c|c|c|}
\hline Source & Sum of Squares & $\mathbf{d f}$ & Mean Square & F Value & p-value (Prob> F) \\
\hline Model & 0.310919 & 5 & 0.062184 & 28.44276 & 0.0002 \\
\hline Pure error & 0.01044 & 4 & 0.00261 & & \\
\hline $\mathrm{R}^{2}$ & 0.9531 & & & & \\
\hline Adj. $\mathrm{R}^{2}$ & $91.96 \%$ & & & & \\
\hline
\end{tabular}

Table 2 Analysis of Variance (ANOVA) for optimization of synthesis of MFeNPs 

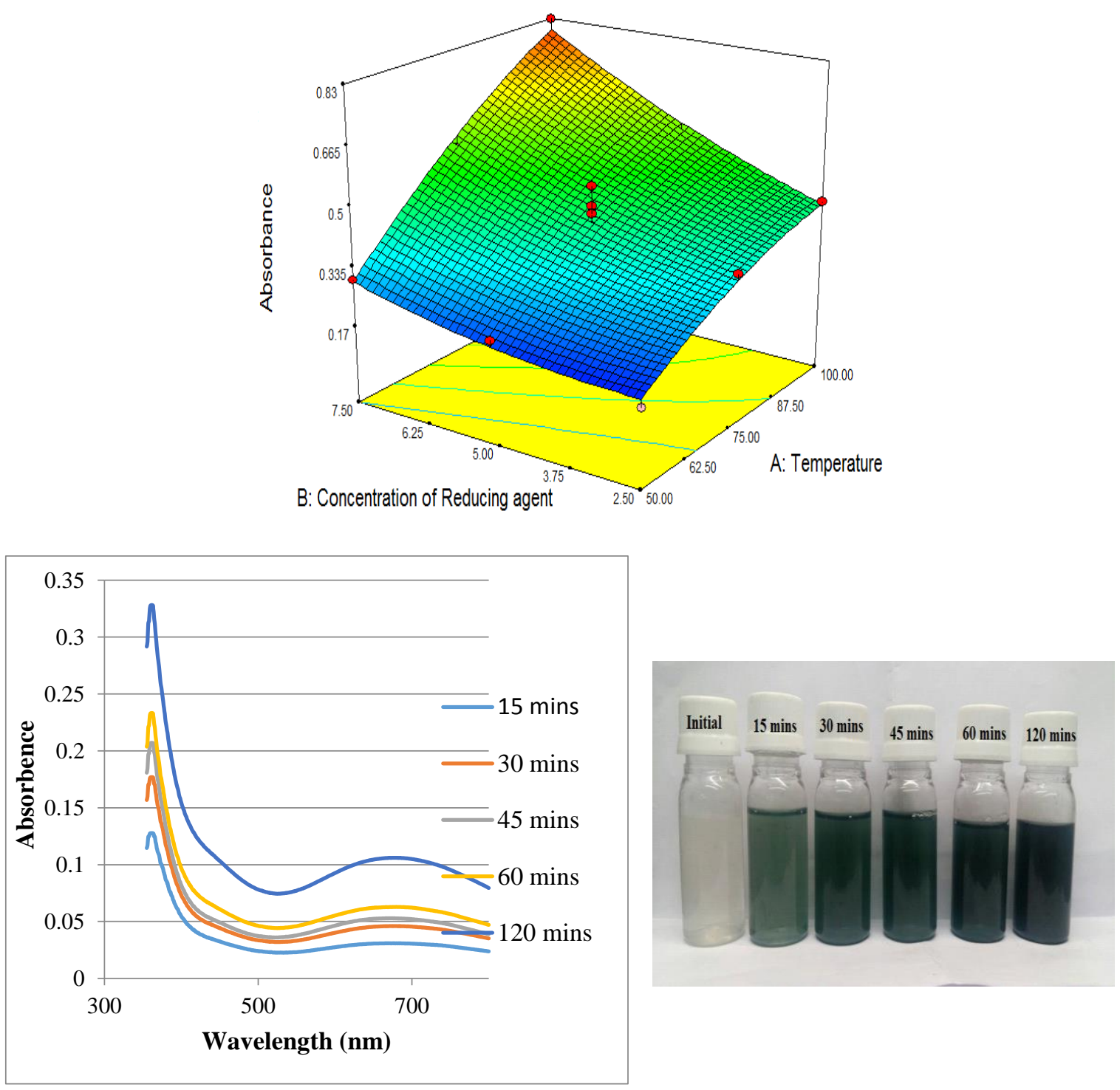

Fig. 1 Response surface plot of Absorbance vs Temperature and concentration of reducing agent Fig. 2 UV-Vis absorption spectrum of MFeNPs solutions at different time intervals. Fig. 3 Images of MFeNPs solutions at different time intervals.

The average particle size was calculated by Debey-Scherrer's formula,

$$
D=\frac{0.94 \lambda}{\beta \cos \theta}
$$

Where $\lambda$ is $X$-ray wavelength $(0.15406 \mathrm{~nm}), \beta$ is full width at half maximum (FWHM) of the diffraction peak in radians, $\Theta$ is Bragg's diffraction angle respectively. The particle size obtained was $61.94 \mathrm{~nm}$. (Prabhu et al. 2015)
The calculated particle size by the XRD, TEM and Dynamic Light Scattering technique are listed in Table 3. The differences in the particle size for these techniques may be due to the aggregation during the sample preparation. (Liu \& Hurt 2010)

\section{Energy Dispersive X-Ray analysis}

Fig. 5 shows the Energy-Dispersive Absorption Spectroscopy of derived MFeNPs, which confirmed the presence of elemental iron by the signals in the range of 6 to $6.5 \mathrm{keV}$. (Muhammad et al. 2016) 


\section{Fourier transforms infrared spectroscopy}

FTIR measurements were carried out to identify the possible bio-molecules responsible for the reduction of ferrous sulphate and capping of the reduced MFeNPs (Fig. 6). The banana leaves and the synthesized MFeNPs were subjected to FTIR that showed various bands, the $\mathrm{O}-\mathrm{H}$ stretching around $3400 \mathrm{~cm}^{-1}$ shows the presence of hydroxyl groups from the polyols such as flavones, terpenoids and polysaccharides present in the leaf extract. The bands at $1645 \mathrm{~cm}^{-1}$ and $1041 \mathrm{~cm}^{-1}$ denotes the presence of organic material in the sample majorly contributed by banana leaves. The rational decrease in intensity of $\mathrm{O}-\mathrm{H}$ stretching might be due to interaction of nanoparticles. These bands confirmed the presence of compounds like flavonoids and terpenoids and hence may be held responsible for efficient capping and stabilization of obtained magnetite nanoparticles. (Ting et al. 2014; Balamurughan et al. 2014)

\section{Crystallite size (nm)}

\begin{tabular}{c|c|c}
\hline $\begin{array}{c}\text { Debey- } \\
\text { Scherrer } \\
\text { formula }\end{array}$ & $\begin{array}{c}\text { Dynamic Light } \\
\text { Scattering } \\
\text { technique }\end{array}$ & TEM \\
\hline 61.94 & 31 & 72 \\
\hline
\end{tabular}

Table 3 Particle size of MFeNPs calculated by different methods a
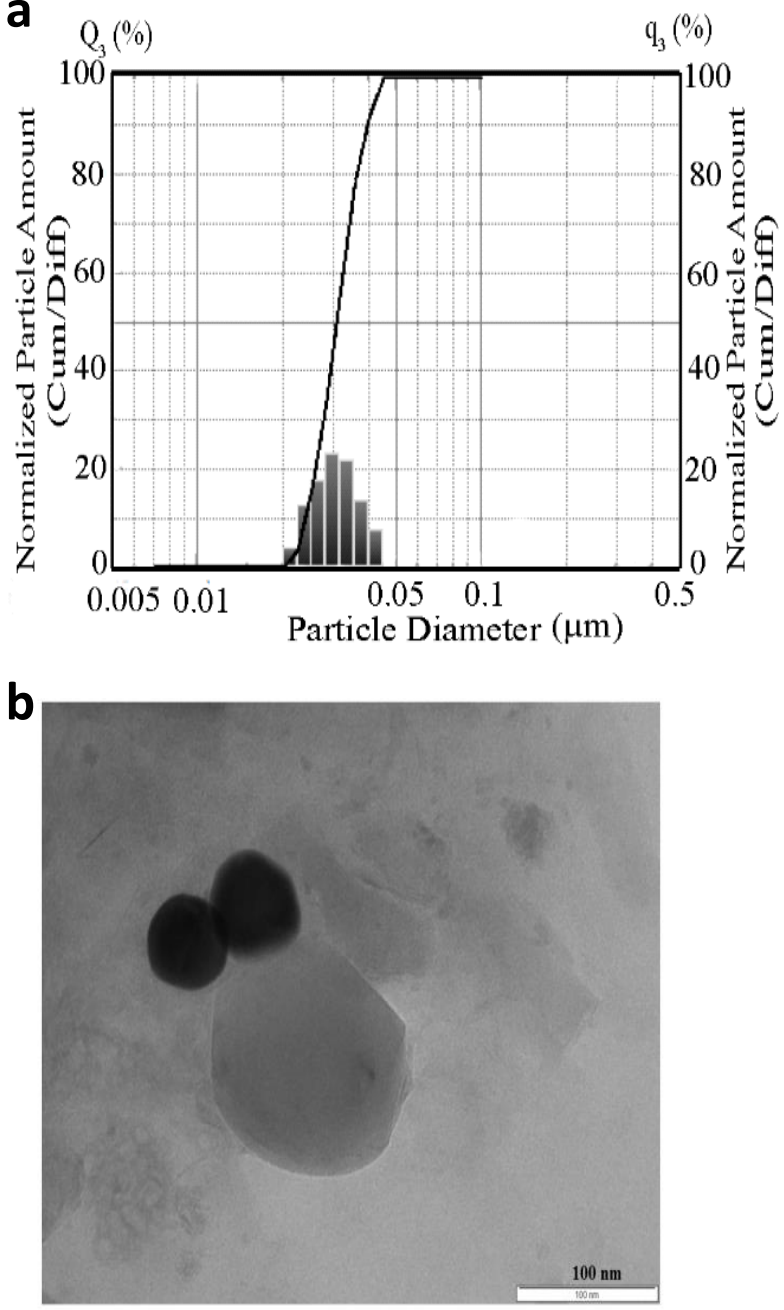

C

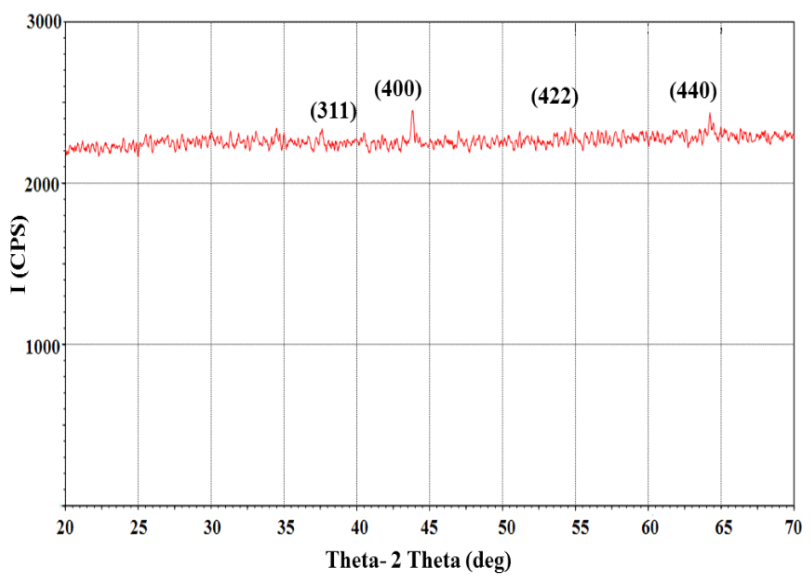

Fig. 4 a. Particle size of MfeNPs. b. TEM image of MfeNPs c. XRD pattern of MfeNPs 


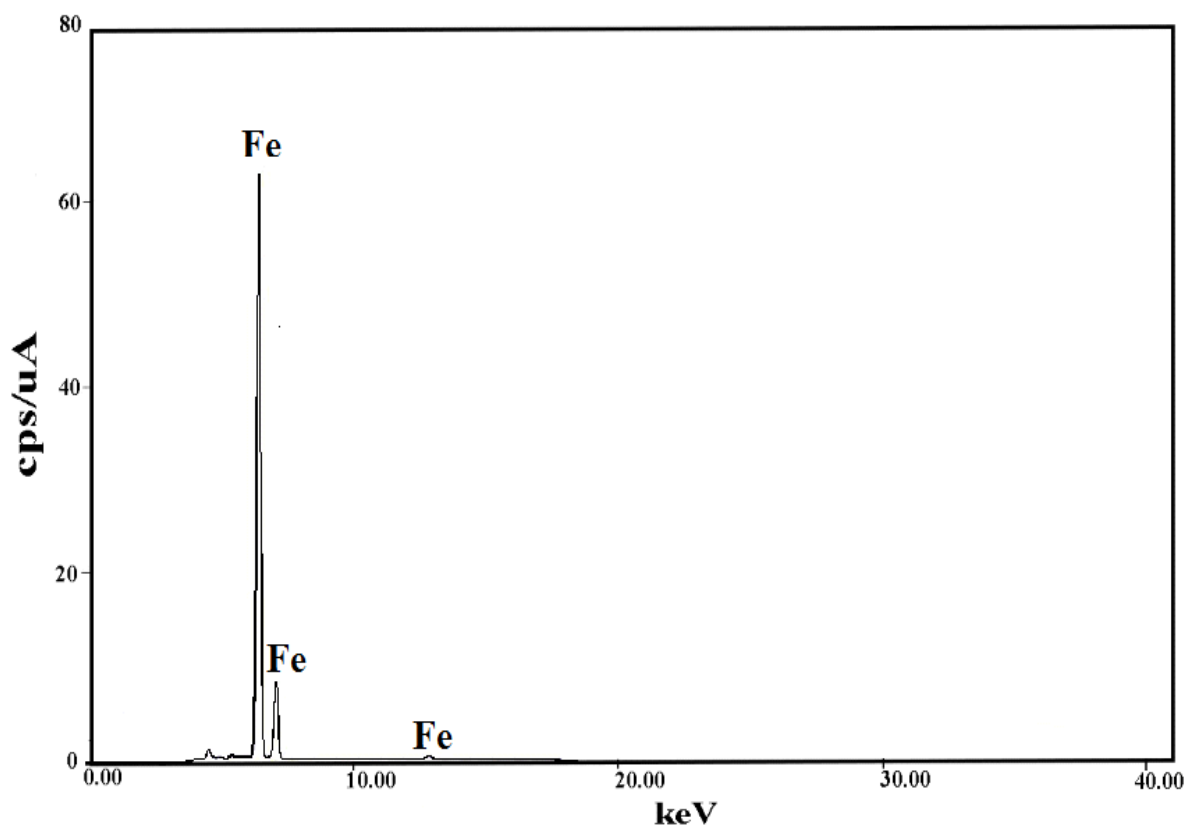

Fig. 5 EDX pattern of synthesized MFeNPs

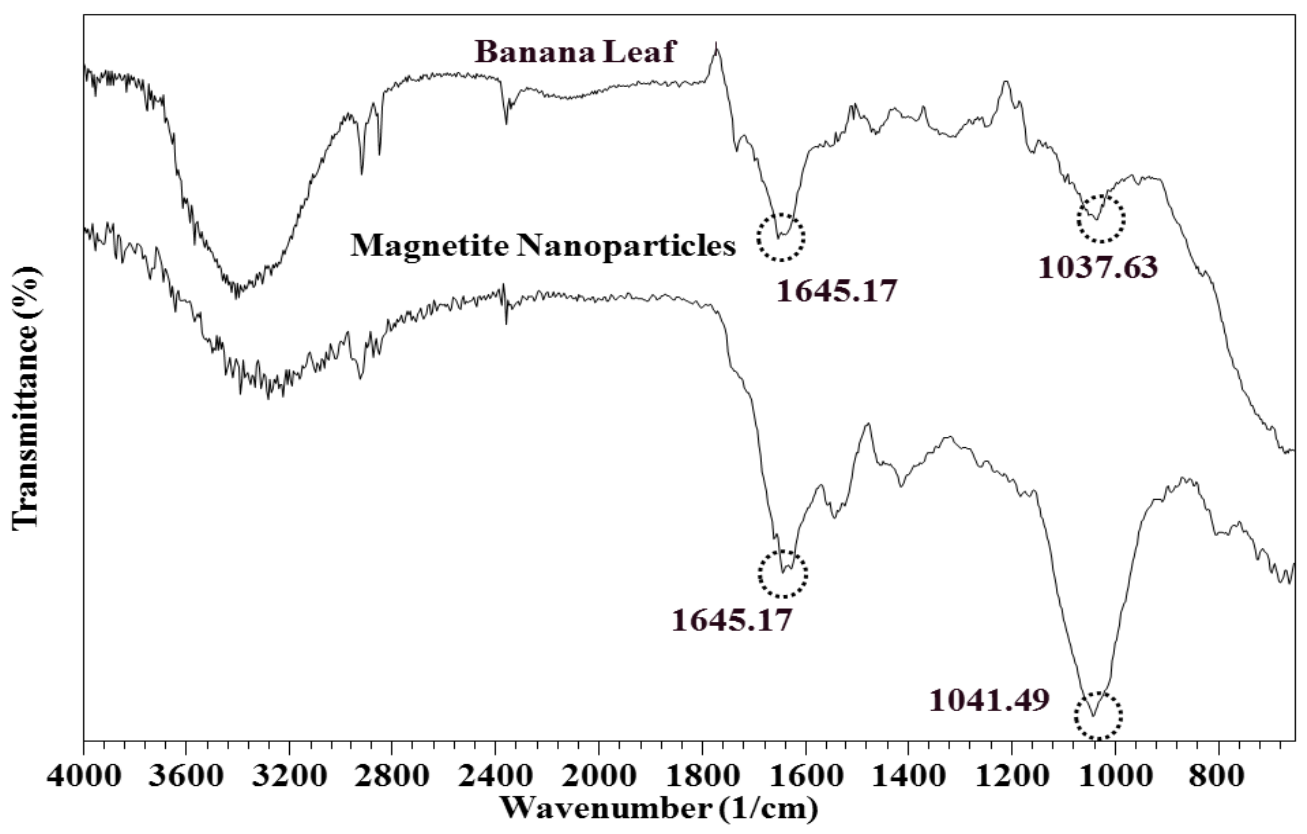

Fig. 6 FTIR spectrum of banana leaf and magnetite nanoparticles

\section{MATERIAL AND METHODS}

\section{Materials}

Ferrous sulphateheptahydrate $\mathrm{FeSO}_{4} .7 \mathrm{H}_{2} \mathrm{O}$ (Mol. Wt. 278.01) and acetic acid of analytical grade were obtained.

\section{Synthesis of MFeNPs}

Banana leaves collected from the institute campus were washed with distilled water and cut into small pieces. $100 \mathrm{ml}$ of $0.1 \mathrm{M}$ aqueous solutions of ferrous sulfate was prepared in a conical flask using distilled water and then $\mathrm{pH}$ 3 was adjusted with the help of acetic acid. In this salt solution, banana leaves were added while stirring continuously on a shaker bath machine (RossariLabtech, Mumbai, India) at 70 rpm for two hours. Experiments were carried out according to the Design of Expert 7 to study the effect of two parameters: temperature and concentration of reducing agent on 
absorbance of MFeNPs. Reduction of ferrous sulphate into MFeNPs was observed through change in colour of solution from light yellow to greyish black, confirming the formation of NPs (Latha et al. 2014). After complete reduction of the ferrous sulphate, the solution was filtered through nylon mesh. The residual solution which contained NPs was centrifuged for $15 \mathrm{~min}$ at $12,000 \mathrm{rpm}$ later washed with distilled water and then dried in oven at $80^{\circ} \mathrm{C}$.

\section{Experimental Design}

When the design economy and precise prediction variance are desired the use of second-order designs such as central composite design (CCD) plays vital role. In the present study, central composite design was used to study the effect of independent variables, i.e. temperature and concentration of reducing agent on the response (absorbance of MFeNPs).

\section{Characterization}

UV-Visible spectrophotometer (UV-1800 ENG $240 \mathrm{~V}$, Shimadzu, Japan) was used for the analysis of synthesized MFeNPs periodically as a function of time in the wavelengths ranging from $200-800 \mathrm{~nm}$ with a resolution of $1 \mathrm{~nm}$. The particle size analysis was done using nano particle size analyser (SALD 7500 nano, Shimadzu, Japan). Surface morphology was studied with transmission electron microscopy (Phillips TEM-200 Supertwin STEM, accelerating voltage-200kV, resolution- 0.23 $n m$ ). Crystallographic study of NPs was carried out using X-ray diffractometer (Shimadzu XRD6100, Japan) with CuK $\alpha$ radiation from $40 \mathrm{kV} / 30 \mathrm{~mA}$ using the $2 \theta$ range of $20-70$. Chemical functional group identification on MFeNPs was determined using FTIR (FTIR 8400 S Shimadzu, Japan) in the spectral range of $750-4000 \mathrm{~cm}^{-1}$ and elemental analysis was done in the $\mathrm{Na}-\mathrm{U}$ channel using EDAX (EDX720, Shimadzu, Japan).

\section{CONCLUSION}

MFeNPswere successfully synthesized using banana leaves as a reducing agent. The protein present in banana leaves was responsible for reduction of ferrous sulphate into MFeNPswhich is also present on the surface of nanoparticle and provide stability. The formation of nanoparticles was accompained with change colour of the solution from light yellow to grayish black giving absorbance peak at $360 \mathrm{~nm}$ in UV-visible spectroscopy. For maximum absorbance (0.328339) the optimized conditions were $70.47^{\circ} \mathrm{C}$ temperature and $2.5 \mathrm{gm} / 100 \mathrm{~mL}$ concentration of reducing agent as per CCD. The MFeNPs formed were of predominantly spherical in shape and crystalline in nature with crystallinity of $19.2212 \%$. The average particle size of magnetite nanoparticles was $31 \mathrm{~nm}$, $61.94 \mathrm{~nm}$ and $72 \mathrm{~nm}$ observed with Particle size analyser, XRD and TEM respectively.

\section{ACKNOWLEDGEMENT}

The authors would like to thank the TEQIP-II and DST-FIST for financial support and testing facilities.

\section{REFERENCES}

Balamurughan, M.G., Mohanraj, S., Kodhaiyolii, S., Pugalenthi, V. 2014, 'Ocimum sanctum leaf extract mediated green synthesis of iron oxide nanoparticles: spectroscopic and microscopic studies', J. of Chemical and Pharmaceutical Sci, vol. 4, pp. 201-204.

Basavegowda, N., Magar, K.B.S., Mishra, K., Lee, Y.R. 2014, 'Green fabrication of ferromagnetic Fe3O4 nanoparticles and their novel catalytic applications for the synthesis of biologically interesting benzoxazinone and benzthioxazinone derivatives', New J. Chem, vol. 38, pp. 5415-5420. Basavegowda, N., Mishra, K., Lee, Y. R. 2014, 'Sonochemically synthesized ferromagnetic Fe304 nanoparticles as a recyclable catalyst for the preparation of pyrrolo [3, 4-c] quinoline-1,3-dione derivatives', RSC Adv, vol. 4, pp. 61660-61666.

Chaki, S.H., Tasmira, J.M., Chaudhary, M.D., Tailor, J.P., Deshpande, M.P. 2015, 'Magnetite Fe304 nanoparticles synthesis by wet chemical reduction and their characterization', Adv. Nat. Sci: Nanosci and Nanotechnology, vol. 6, pp. 1-6.

Gawande, M.B.B.P.S. and Varma, R.S. 2013, 'Nanomagnetite (Fe304) as a support for recyclable catalysts in the development of sustainable methodologies', Chem. Soc. Rev, vol. 42, pp. 33713393.

Hamed, B., Soheila, H., Pouneh, E., Milad, M.A., Ahad, A., Farzaneh, N. 2014, 'Microbial mediated preparation, characterization and optimization of gold nanoparticles', Brazilian Journal of Microbiology, vol. 45, pp. 1493-1501. 
Haw, C.Y., Mohamed, F., Chia, C.H., Radiman, S., Zakaria, S., Huang, N.M., Lim, H.N. 2010, 'Hydrothermal synthesis of magnetite nanoparticles as MRI contrast agents', Ceram Int, vol. 36, pp. 1417-1422.

Hu, F.Q., Wei, L., Zhou, Z., Ran, Y.L., Li, Z., Gao, M.Y. 2006, 'Preparation of biocompatible magnetite nanocrystals for in vivo magnetic resonance detection of cancer', Adv. Mater, vol. 18, pp. 25532556.

Kavitha, A.I., Prabhu, H.G., Babu, S.A. Suja, S.K. 2013, 'Magnetite nanoparticles-chitosan composite containing carbon paste electrode for glucose biosensor application', J.Nanosci. Nanotech, vol.13, pp. 98-104.

Kumfer, B.M., Shinoda, K., Jeyadevan, B., Kennedy, I.M. 2010, 'Gas-phase flame synthesis and properties of magnetic iron oxide nanoparticles with reduced oxidation state', J. Aerosol Sci, vol. 41, pp. 257-265.

Latha, N. and Gowri, M., 2014, 'and characterization of $\mathrm{Fe} 3 \mathrm{O} 4$ nanoparticles using Caricaya papaya leaves extract', Int. J. Sci. Res, vol. 3, pp. 1551-1556.

Lemine, O.M., Omri, K., Zhang, B., Mir, E.L., Sajieddine, M., Alyamani, A., Bououdina, M. 2012, 'Sol-gel synthesis of $8 \mathrm{~nm}$ magnetite (Fe304) nanoparticles and their magnetic properties', Superlattice Microst, vol. 52, pp. 793-799.

Li, X.L., Li, H., Liu, G.Q., Deng, Z.W., Wu, S.L., Li, P.H., $\mathrm{Xu}$, Z.S., Xu, H.B., Chu, P.K., 2012, 'Magnetiteloaded fluorine-containing polymeric micelles for magnetic resonance imaging and drug delivery', Biomaterials, vol. 33, pp. 3013-3025.

Liu, J., and Hurt, R.H., 2010, 'Ion release kinetics and particle persistence in aqueous nanosilver colloids', Environ. Sci. Technol. 44(2010), pp. 169-2175'

Mahadavian, A.R. and Mirrahimi, M.A.S. 2010, 'Efficient separation of heavy metal cations by anchoring polyacrylic acid on superparamagnetic magnetite nanoparticles through surface modification', Chem. Eng. J, vol. 159, pp. 264-271.

Muhammad, A.Z., Sonita, A., Deana, W., Mia, L. 2016, 'LedyastutiPreparation of Fe304-chitosan hybrid nano-particles used for humic Acid adsorption', Environmental Nanotechnology, vol. 6, pp. 64-75.

Narayanan, S., Sathy, B.N., Mony, U., Koyakutty, M., Nair, S.V., Menon, D. 2012, 'Biocompatible magnetite/gold nanohybrid contrast agents via green chemistry for MRI and CT bioimaging', ACS Appl. Mater Interfaces, vol. 4, pp. 251-260.

Paiva, D.L., Andrade, A.L., Pereira, M.C., Fabris, J.D., Domingues, R.Z., Alvarenga, M.E. 2015, 'ME Novel protocol for the solid-state synthesis of magnetite for medical practices', Hyperfine Interact, vol. 232, pp. 19-27.
Petla, K., Vivekanandhan, S., Misra, M., Mohanty, A.K., Satyanarayana, N. 2012, 'Soybean (Glycine Max) Leaf Extract Based Green Synthesis of Palladium Nanoparticles', J. Biomat. Nanobiotechnol, vol. 3, pp. 14-19.

Prabhu, Y.T., VenkateswaraRao, K., SeshaSai, V., Tambur, P. 2015, 'A facile biosynthesis of copper nanoparticles: A micro-structural and antibacterial activity Investigation', J. of Saudi Chemical Society, vol.21, pp. 180-185.

Priest, S. 2006, 'The North American opinion climate for nanotechnology and its products: opportunities and challenges', J Nanopart, vol. 8, pp. 563-568.

Qiao, R.R., Yang, C.H., Gao, M.Y. 2009, 'Superparamagnetic iron oxide nanoparticles: from preparations to in vivo MRI applications', J. Mater. Chem, vol. 19, pp. 6274-6293.

Salem, M., Xia, Y., Allan, A., Rohani, S., Gillies, E.R. 2015, 'Curcumin-loaded, folic acid-functionalized magnetite particles for targeted drug delivery', RSC Adv, vol. 5, pp. 37521-37366.

Shankar, S.S., Rai, A., Ankamwar, B., Singh, A., Ahmad, A., Sastry, M. 2004, 'Biological synthesis of triangular gold nanoprisms', Nat. Mater, vol. 3 pp. 482-487.

Sharad, N.S., Swapnil, R.B., Ganesh, R.M., Samadhan, S.K., Dinesh, K.M., Shashikant, B.B., Anuj, K.R., Nenad, B., Orlando, M.N.D.T., Radek, Z., Rajender, S.V., Manoj, B.G. 2014, 'Iron oxidesupported copper oxide nanoparticles (nanocat-Fe$\mathrm{CuO}$ ): magnetically recyclable catalysts for the synthesis of pyrazole derivatives, 4-methoxyaniline, and ullmann-type condensation reactions', ACS Sustainable Chem. Eng, vol. 2, pp. 69-75.

Senthil, M. and Ramesh, C. 2012, 'Synthesis of Fe3O4 nanoparticles using Tridaxprocumbens leaf extract and its antibacterial activity on pseudomonas aeruginosa', J. Nanomater Biostruct, vol. 7, pp. 1655-1659.

Ting, W., Jiajiang, L., Zuliang, C., Mallavarapu, M., Ravendra, N., 2014, 'Green synthesized iron nanoparticles by green tea and eucalyptus leaves extracts used for removal of nitrate in aqueous solution, Journal of Cleaner Production', J. of Cleaner Production, vol. 7, no. 83, pp.413-419.

Terris, B.D. and Thomson, T. 2005, 'Nanofabricated and self-assembled magnetic structures as data storage media', J Phys D Appl. Phys, vol. 38, pp. 1-9. Venkateswarlu, S., Rao, Y.S., Balaji, T., Prathima, B., Jyothi, N.V.V. 2013, 'Biogenic synthesis of Fe3O4 magnetic nanoparticles using plantain peel extract', Mater Lett, vol. 100, pp. 241-248.

Wani, K.D., Kadu, B.S., Mansara, P., Gupta, P., Deore, A.V., Chikate, R.C., Poddar, P., Dhole, S.D., Kaul-Ghanekar, R. 2014, 'Synthesis, 
characterization and in vitro study of biocompatiblecinnamaldehyde functionalized magnetite nanoparticles (CPGF Nps) for hyperthermia and drug delivery applications in breast cancer', Plos One, vol. 9, pp. 1-13.

Yen, P.Y., Kamyar, S., Mikio, M., Noriyunki, K., NurulBahiyah, B.A.K., ShazaBtMohamad, E., Kar, L.X. 2016, 'Green Synthesis of Magnetite (Fe304) Nanoparticles Using Seaweed (Kappaphycusalvarezii) Extract', Nanoscale Research Letters, vol. 11, pp. 276-284.

Zargar, M., Hamid, A.A., Bakar, F.A., Shamsudin, M.N., Shameli, K., Jahanshiri, F., Farahani, F. 2011, 'Green synthesis and antibacterial effect of silver nanoparticles using Vitexnegundo L. Molecules', Molecules, vol. 16, pp. 6667-6676.

Zhang, L., Dong, W.F., Sun, H.B. 2013, 'Multifunctional superparamagnetic iron oxide nanoparticles: design, synthesis and biomedical photonic applications', Nanoscale, vol. 5, pp. 76647684.

Zhao, H., Saatchi, K., Hafeli, U.O. 2009, 'Preparation of biodegradable magnetic microspheres with poly (lactic acid)-coated magnetite', J. Magn. Mater, vol. 321, pp. 1356-1363. 